\title{
Multi-objective parametric study of a wooden window
}

\author{
Klodian Gradeci ${ }^{1, *}$, Nathalie Labonnote ${ }^{1}$, and Steinar Grynning ${ }^{1}$ \\ ${ }^{1}$ SINTEF Building and Infrastructure, Trondheim, Norway
}

\begin{abstract}
The aim of this study is to conduct a multi-objective parametric study by simultaneously analysing the thermal and structural performance of a wood-frame window. The finite element simulation program COMSOL is used to carry out the thermal and structural analysis. A global sensitivity analysis is performed to screen and rank the dominant parameters. Afterwards, a parametric analysis is performed by varying the dominant parameters. The results demonstrate that the performance of the frame configuration of the wooden window can be improved from the nominal configuration. The results suggest that the methodology can be further improved by conducting form-finding and typology-based optimization studies while accounting further for the impact of the window on the energy use for heating in buildings.
\end{abstract}

\section{Introduction}

The overall geometry and design of wooden window frames have changed little in the last decades. In a 40-50-year perspective, the major change that can be observed is that frame dimensions have been increased. This is likely due to increased structural stresses following the need to accommodate thicker, multi-layer glazing units. Therefore, research has mostly been focused on improving the thermal performance of the window while research related to optimization of structural and thermal properties and to the resulting performance of window frames is scarce. The aim of this study is to develop a multi-objective parametric study by analysing the thermal and structural performance of a window frame. The following objectives are addressed:

- Perform a global sensitivity analysis to find the most dominant geometrical dimensions of the frame that influence the thermal and structural performance of the window.

- Perform a parametric analysis to investigate the relation of these dominant parameters and exploit the feasibility of window frame optimization.

Section 0 carries out a state-of-the-art review involving optimisation of window constructions or related topics. Section 0 presents the window configuration with the varying parameters and the methods used for the sensitivity and parametric analysis. Section 0 presents and discusses the results. Section 0 draws the conclusions and discusses further research.

* Corresponding author: klodian.gradeci@sintef.no 


\section{Literature review: State of the art}

An analysis of the available literature shows that only a few studies could be found that investigate the optimization of windows. The studies range within the timeframe of 20102017 and are presented in a tabular format below. The review shows that optimization of the window has been discussed during the past years. However, it must still be considered a topic under research. Most of the literature focuses on discrete variation of the window configurations and optimization based on their thermal performance. While the contribution of the frame (and its material) to the decrease of the thermal performance of windows has been widely discussed, only one publication has investigated the structural performance of frame. Similarly, only one study investigates the effect of different geometrical variables of the frame of the window while all the other studies focus on varying the configuration of the window and its material.

Table 1. Overview of the literature. ${ }^{\dagger}$

\begin{tabular}{|c|c|c|c|c|c|c|c|c|}
\hline Literature & \multicolumn{2}{|c|}{ Type of method } & \multicolumn{3}{c|}{ Type of approach } & \multicolumn{3}{c|}{ Type of analysis } \\
\hline & Optimisation & Parametric & Geometry & Form finding & Typology & Thermal & Structural & Visual \\
\hline$[1]$ & & $\checkmark$ & & & $\checkmark$ & & $\checkmark$ & \\
\hline$[2]$ & & $\checkmark$ & & & $\checkmark$ & $\checkmark$ & & $\checkmark$ \\
\hline$[3]$ & & & & & & & & \\
\hline$[4]$ & & $\checkmark$ & $\checkmark$ & & $\checkmark$ & $\checkmark$ & & \\
\hline$[5]$ & $\checkmark$ & & $\checkmark$ & $\checkmark$ & $\checkmark$ & $\checkmark$ & & \\
\hline$[6]$ & & $\checkmark$ & & & $\checkmark$ & $\checkmark$ & & \\
\hline$[7]$ & $\checkmark$ & & $\checkmark$ & & $\checkmark$ & $\checkmark$ & & \\
\hline$[8]$ & & $\checkmark$ & $\checkmark$ & & & $\checkmark$ & & \\
\hline
\end{tabular}

\section{Materials and methods}

\subsection{Description of the wooden window}

The selected window is of a standard size of $1.23 \mathrm{~m} \times 1.48 \mathrm{~m}$ (see Fig. 1, Table 2 and Table 3 ) and made with a wooden frame with a traditional two-pane insulated glazing unit (IGU). The window frame is selected from the list provided in the standard ISO 10077-2:2017 [9] for the validation of calculation programs.

\footnotetext{
${ }^{\dagger}$ Method includes: a) Optimisation: a method that searches the design space efficiently and finds the optimum solution; and b) Parametric: a method that investigates the influence of different geometric or physical parameters or both on the solution of the problem. Approach includes: a) Geometry: geometrical dimensional and/or ratios; b) Form finding: the form of the geometry is searched by varying its geometry as continues variables; and, c) Typology: geometrical components and materials are varied. Analysis includes: a) Thermal; b) Structural; and c) Visual.
} 

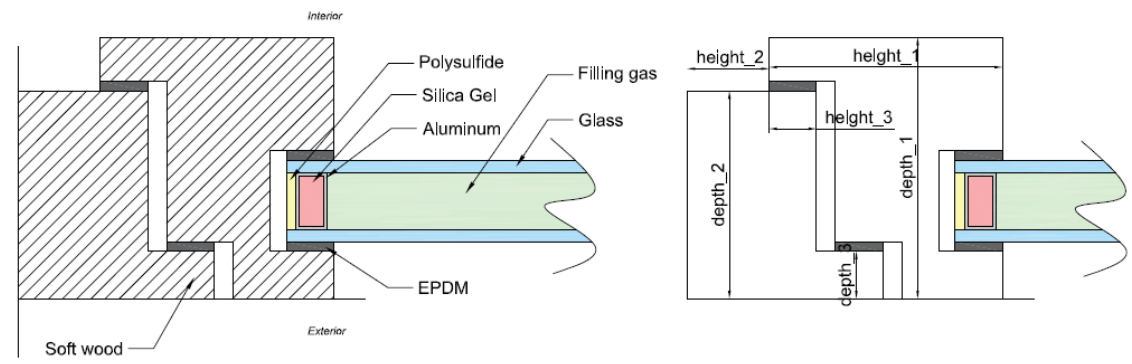

Fig. 1. Geometry of the wood frame with a glazing as taken from ISO 10077-2:2017 [9]

Table 2. Nominal and parametric dimensions of the window frame.

\begin{tabular}{|c|c|c|c|}
\hline Parameter & Description & $\begin{array}{c}\text { Nominal } \\
\text { value [mm] }\end{array}$ & $\begin{array}{c}\text { Range } \\
{[\mathrm{mm}]}\end{array}$ \\
\hline depth_1 & Depth of the sash & 83 & {$[71: 89]$} \\
\hline depth_2 & Depth of the frame & 66 & {$[58: 72]$} \\
\hline depth_3 & Depth of right part of frame & 15 & {$[13: 19]$} \\
\hline height_1 & Height of the sash & 84 & {$[72: 90]$} \\
\hline height_2 & Height of the left part of frame & 26 & {$[18: 30]$} \\
\hline height_3 & Height of the right part of frame & 16 & {$[13: 19]$} \\
\hline
\end{tabular}

Table 3. Properties for the materials used in the numerical model.

\begin{tabular}{|c|c|c|c|}
\hline Material & $\begin{array}{c}\text { Density } \\
{\left[\mathrm{kg} / \mathrm{m}^{3}\right]}\end{array}$ & $\begin{array}{c}\text { Young's modulus } \\
{[\mathrm{GPa}]}\end{array}$ & $\begin{array}{c}\text { Thermal Cond. } \\
{[\mathrm{W} /(\mathrm{m} \cdot \mathrm{K})]}\end{array}$ \\
\hline Soft wood & 500 & 11 & 0,13 \\
\hline Glass & 2500 & 70 & 1 \\
\hline EPDM & 1150 & 0,006 & 0,035 \\
\hline Aluminium & 2800 & 68,3 & 160 \\
\hline Silica gel & 720 & 1,85 & 0,13 \\
\hline Polysulfide & 1150 & 2,5 & 0,4 \\
\hline
\end{tabular}

\subsection{Methodology}

\subsubsection{General overview}

A schematic overview of the methodology is presented in Fig. 2. Six geometrical parameters (see Table 2) are selected to be varied. The details are described in the following paragraphs and sections.

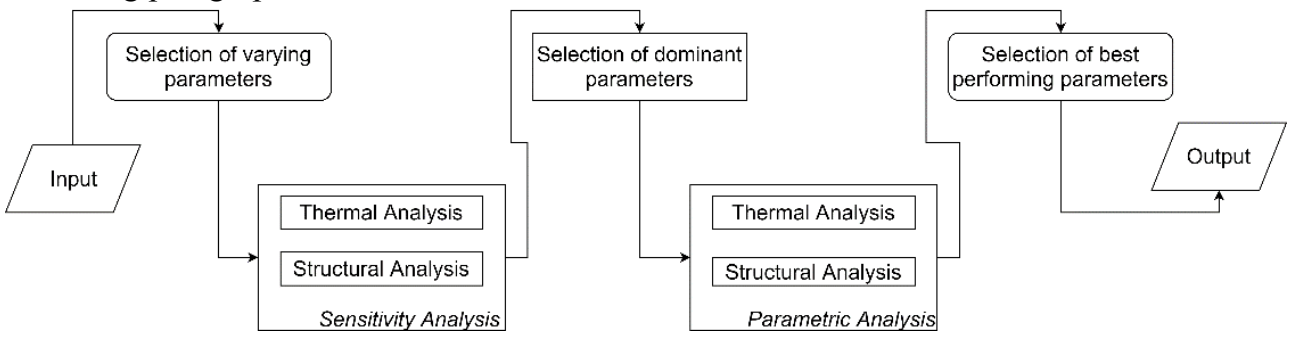

Fig. 2. Schematic overview of the methodology. 
Modelling assumptions: The wooden window frame is modelled and validated according to the international standard EN ISO 10077-2 [9] using the method for the treatment of cavities. The reader is referred to the standard for further details regarding the calculation details, modelling assumptions, boundary conditions.

Thermal analysis: The finite element simulation program COMSOL [10] is used to perform the thermal analysis. It uses heat transfer coefficients prescribed by EN ISO 10077-2 [9] to solve the conductive heat transfer equations in the window frame. A twodimensional analysis is performed. Two parameters are selected to study the thermal performance (output variable). The thermal conductance of the entire section $L^{2 D}$ is calculated according to:

$$
L^{2 D}=\frac{\varnothing}{T_{e}-T_{i}}
$$

where $\varnothing$ is the heat flow rate through the window (in $\mathrm{W} / \mathrm{m}$ ), $T_{e}=0{ }^{\circ} \mathrm{C}$ is the external temperature and $T_{i}=20^{\circ} \mathrm{C}$ is the internal temperature.

The linear thermal transmittance of the frame $\Psi$ defined by:

$$
\Psi=L^{2 D}-U_{f} b_{f}-U_{g} b_{g}
$$

where $U_{f}$ is the thermal transmittance of the frame, $b_{f}$ is the projected width of the frame sections, $b_{g}$ is the visible width of the glazing, $U_{g}$ is the thermal transmittance of the central area of the glazing.

Structural analysis: The structural analysis was based on the load capacity of maximum displacement (output variable). A three-dimensional FEM was built up for this simulation in COMSOL. A pressure load of $\mathrm{P} 1=600 \mathrm{~Pa}$ was applied to the external part of the window by following the guidelines for experimental set-ups provided in EN ISO 10077-1 [9] and in SINTEF Certification [11]. A convergence analysis was performed for the mesh detailing. The two- and three-dimensional finite element models of the window are shown in Chyba! Nenalezen zdroj odkazů..

\subsubsection{Sensitivity analysis}

The Morris method [12] is selected as a method to carry out the global sensitivity analysis. It varies one parameter at a time and screens important or negligible parameters. The method calculates two sensitivity measures for each parameter: a) the mean $\mu$ indicating the overall effect of the parameter and $b$ ) the standard deviation $\sigma$ indicating either interaction with other parameters or non-linear behaviour. A total number of 6 parameters are considered for this study (see Table 2). Therefore, a total of $(6+1) \times 10=70$ simulations are performed. SimLab [12] is used to sample the variables and calculate the sensitivity measures after all simulations are computed in COMSOL. The disadvantage of Morris method is that it is only a screening method, and therefore, does not reveal the extent of the influence of each parameter.

\subsubsection{Parametric analysis}

A parametric analysis is carried out with the screened dominant parameters for the thermal and structural performance of the window. The parametric analysis has been performed within the COMSOL model by assigning the specific values to each varying parameter. 


\section{Results and Discussion}

\subsection{Sensitivity analysis}

The results of the global sensitivity analysis are presented schematically in Fig. 3 and the ranking and selection of dominant parameters (in grey colour) are displayed in Table 4.

Table 4. Ranking of the most dominant parameters.

\begin{tabular}{|c|c|c|}
\hline Parameter & \multicolumn{2}{|c|}{ Ranking according to performance } \\
\hline & Thermal Performance & Structural Performance \\
\hline depth_1 & 2 & 3 \\
\hline depth_2 & 1 & 4 \\
\hline depth_3 & 6 & 6 \\
\hline height_1 & 5 & 1 \\
\hline height_2 & 4 & 5 \\
\hline height_3 & 3 & 2 \\
\hline
\end{tabular}

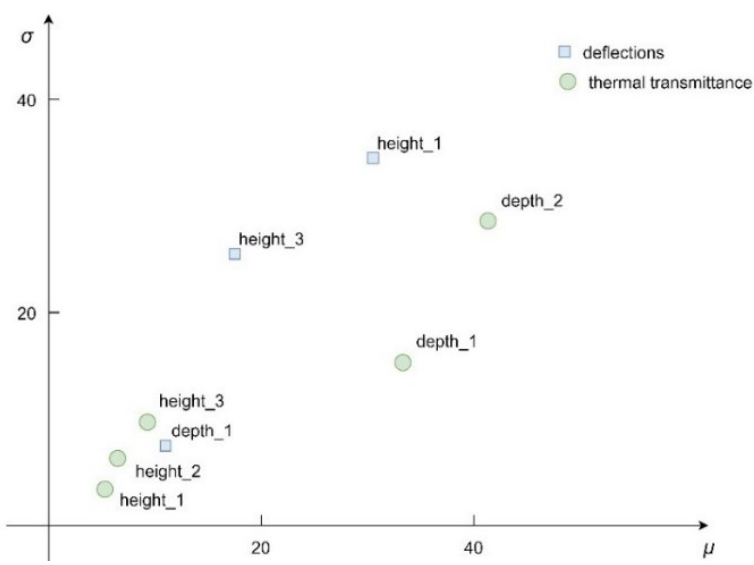

Fig. 3. Sensitivity analysis results showing only the influential parameters.

The results show that there are five dominant parameters for the thermal performance and three dominant parameters for the structural performance. The latter implies that the geometry of the window frame can be optimized by modifying the non-dominant parameters. This can be achieved by adjusting the geometric dimensions that were found to be non-dominant for the structural analysis and dominant for the thermal analysis.

\subsection{Parametric analysis: Thermal and Structural Analysis}

The sensitivity analysis suggested that five parameters are dominant for the joint performance (thermal and structural) of the window frame. Consequently, a parametric analysis was carried out by varying these five parameters. Since there are two different criteria for the best performing configuration: thermal and structural performance, two different best performing, or optimal, solutions are found based on how they minimise the objective functions defined in section 0 . The geometric characteristics of these solutions are presented in Table 5. The relative change in terms of structural and thermal performance is provided graphically in Fig. 4. 
Table 5. Geometric characteristics of the nominal configuration and two found optimal solutions.

\begin{tabular}{|c|c|c|c|c|c|}
\hline Configuration & \multicolumn{5}{|c|}{ Parameters [mm] } \\
\hline & depth_1 & depth_2 & height_1 & height_2 & height_3 \\
\hline Nominal & 83 & 60 & 84 & 26 & 19 \\
\hline Optimal 1 (thermal) & 87 & 69 & 72 & 18 & 19 \\
\hline $\begin{array}{c}\text { Optimal 2 } \\
\text { (structural) }\end{array}$ & 85 & 64 & 89 & 36 & 15 \\
\hline
\end{tabular}

The first optimal solution is found based on the configuration with the best structural performance. It can be observed that there is only a small increase in the structural performance; however, the thermal performance is also increased. The second optimal solution is found based on the configuration with the best thermal performance. It can be observed a large increase in the thermal performance and a large decrease on the structural performance. However, due to the lack of standards/codes regarding Service Limit States for the performance of windows, it cannot be concluded whether the found values meet the necessary requirements for a safe application. Both optimal solutions are found to be better performing in terms of thermal performance. This implies that there is a possibility for performing an optimization study and finding the optimum of the solutions. It should be taken into account that the two identified solutions are based on the discrete samples built up by different combination of the preselected geometric parameters and their variations.

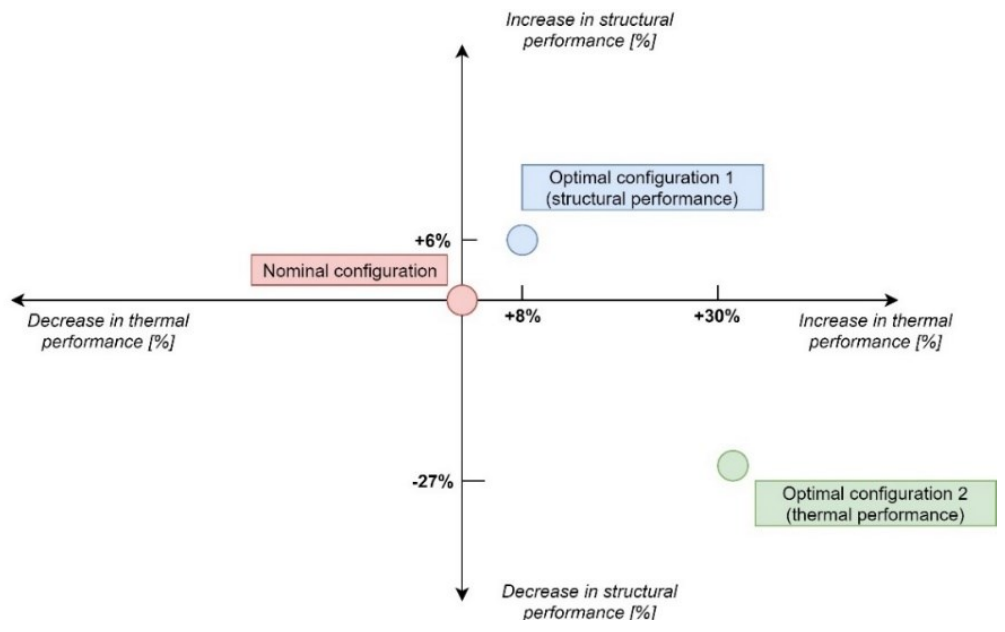

Fig. 4. Difference of performance between the nominal configuration and two optimal solutions.

\section{Conclusion}

The aim of this study was to conduct a multi-objective parametric study by simultaneously analysing the thermal and structural performance of a wooden window. The results demonstrated that the performance of the wooden frame of the window could be improved by performing sensitivity and parametric analysis. The result suggest that the methodology can be further improved by conducting form finding and typology optimization while accounting further for the impact of the window on the energy use for heating in buildings. 
The authors gratefully acknowledge the support from the Research Council of Norway and the industry partners Røros Dør og Vindusfabrikk and Isola through the research project "Highly insulating windows with integrated technology - HVIT" under grant No. 282351.

\section{References}

1. D. Appelfeld, C. S. Hansen, and S. Svendsen, E\&B, 42, 10, (2010)

2. A. Gasparella, G. Pernigotto, F. Cappelletti, and P. Baggio, E\&B, 43, (2011)

3. S. Jaber and S. Ajib, E\&B, vol. 43, no. 11, pp. 3208-3215, (2011)

4. J. Zajas and P. Heiselberg, in Building Simulation, (2011)

5. J. Zajas and P. Heiselberg, in Building Simulation, 7, 6, (2014).

6. N. Van Den Bossche, L. Buffel, and A. Janssens, 78, (2015)

7. J. Ferdyn-Grygierek and K. Grygierek, Energies, 10,10 (2017)

8. P. Janssen, P. Loh, A. Raonic, and M. Schnabel, CAADRIA, (2017)

9. E. ISO, "10077-2 (2017)

10. A. Comsol, (2005)

11. S. Byggforsk, "Vinduer av tre," Sintef Byggforsk, (2002)

12. A. Saltelli et al., Global sensitivity analysis: the primer. (2008) 